\title{
Principales estrategias en la obtención de poliploides en moluscos bivalvos
}

\author{
Main strategies to obtain polyploids in bivalve molluscs
}

Varela A, Alfaro-Mora R. Principales estrategias en la obtención de poliploides en moluscos bivalvos. Rev Colombiana Cienc Anim. Recia. 2020; 12(2):e844. https://doi.org/10.24188/recia.v12. $\mathrm{n} 2.2020 .844$

Universidad de Sucre, Colombia

Los autores permiten a RECIA reimprimir el material publicado en él. En caso de que un autor quiera traducir o usar una publicación parcial o completa de nuestro Diario, el autor debe obtener un permiso por escrito del editor de la revista.

Copyright (C) 2020. El (los) autor (es). Este es un artículo de acceso abierto distribuido bajo los términos de Creative Commons Attribution 4.0 (https://creativecommons.org/licenses/by-ncsa/4.0/), El uso, distribución o reproducción está permitido, siempre que se acrediten al autor original y al propietario del copyright y que se cite la publicación original en esta revista, de acuerdo con la práctica académica aceptada. No se permite el uso, distribución o reproducción que no cumpla con estos términos. 


\title{
Principales estrategias en la obtención de poliploides en moluscos bivalvos
}

\author{
Main strategies to obtain polyploids in bivalve molluscs
}

\author{
Alexander Varela M. Ing. \\ Laboratorio Sea Farmers. Consultor en sanidad acuícola. Sinaloa, México \\ alexander.varela@gmail.com \\ id https://orcid.org/0000-0002-5642-2160
}

Ramsés Alfaro-Mora. M.Sc.

Universidad Latina de Costa Rica, San José, Costa Rica ramses.alfaro@ulatina.net

(D) https://orcid.org/0000-0002-7331-9405
DOI: https://doi.org/10.24188/recia.v13.n1.2021.844

\section{RESUMEN}

El crecimiento en la producción de moluscos se atribuye principalmente al aumento de las especies cultivadas, a la expansión de las regiones geográficas de producción y al mejoramiento de las técnicas productivas. Las líneas cultivadas han sido sometidas a estudios rigurosos sobre sus sistemas biológicos, su potencial y mejora genética. Entre las estrategias utilizadas, el uso de poliploides ha permitido grandes avances zootécnicos, al mismo tiempo que ha representado grandes desafíos técnicos. El presente trabajo describe las principales técnicas de inducción a poliploidías, así como sus limitantes y desafíos. Se describen los métodos físicos y químicos de inducción y sus principales características y resultados obtenidos.

Palabras clave: Bivalvos; cromosomas; inducción; mecanismos; mejoramiento; poliploides.

\begin{abstract}
Global production of bivalve has grown greatly due mainly to the increase in cultivated species, the expansion of geographical production regions and the improvement of production techniques. The lines of cultivated organisms have undergone rigorous studies on their biological systems, their potential and genetic improvement. Among the strategies used, the use of polyploids has allowed great zootechnical advances, while having represented great technical challenges. This work describes the main techniques of induction to polyploidies, as well as their limiters. It describes the physical and chemical methods of induction and their main characteristics and results obtained.
\end{abstract}

Keywords: Bivalve; chromosome; improvement; induction; mechanisms; polyploid. 


\section{INTRODUCCIÓN}

La producción global de moluscos bivalvos ascendió a 17,1 millones de toneladas durante el 2016, logro debido, en gran parte, a los avances en los sistemas de producción utilizados (1), así como a la ampliación de regiones geográficas de cultivo (2).

Consecuentemente, se ha generado un creciente interés en el desarrollo de los conocimientos zootécnicos necesarios para la producción de sus diferentes especies, con constantes avances y publicaciones sobre aspectos como fisiología, alimentación, condiciones de crecimiento, reproducción y prácticas de producción $(3,4,5,6,7)$.

Unido a lo anterior, la expansión de las zonas de cultivo y la introducción de especies en áreas ajenas a su región geográfica original ha exigido el mejoramiento de las líneas cultivadas, con el fin de permitir la adaptación de estas a entornos climatológicamente diferentes (8), mejoramiento que ha requerido de estudios de adaptabilidad y tolerancia $(4,8,9)$.

Entre las diferentes especies de bivalvos que han sido introducidas en América, destaca la ostra japonesa (Crassostrea gigas) por el incremento en su producción, que ha originado su expansión geográfica a países como Estados Unidos, Canadá, México, Guatemala, El Salvador, Honduras, Nicaragua, Costa Rica, Panamá, Colombia, Ecuador, Perú y Chile (2), no descartándose que otros países de América también adopten su cultivo.

Los orígenes del cultivo de C. gigas en América se remontan a la década de 1970 en México, actualmente esta especie es la más cultivada en el noreste del Golfo de California $(9,10,11)$.

Las técnicas de cultivo utilizadas para su producción acuícola se conforman por diferentes etapas que incluyen la producción de semilla, crianza en zonas costeras, cosecha y comercialización $(12,13)$.

La producción de semilla abarca diversas estrategias de mejora, que incluyen manejo de alimentación y temperatura en laboratorios $(14,15)$; mientras que la cosecha y comercialización exige protocolos que garanticen una buena calidad e inocuidad de los productos $(4,12)$.

Estas condiciones, razonablemente controladas en los laboratorios y en procesos post cosecha, contrastan con las presentes en las zonas de crianza, donde no es posible controlar los parámetros (7), debido a una total dependencia de las mareas y corrientes naturales, imposibilitando su control, ajuste o modificación. Consecuentemente, se enfrenta el desafío de disponer de organismos capaces de soportar condiciones ambientales fuera de sus rangos naturales $(8,13)$.

Se han propuesto diferentes estrategias, incluyendo el mejoramiento genético (16), el desarrollo de híbridos entre especies cercanas $(17,18,19,20)$ y el desarrollo de poliploides $(5,6,8,18,21)$. Esta última opción ha resultado particularmente factible en bivalvos, dadas sus particularidades reproductivas.

Por ello, se han desarrollado técnicas de producción de organismos poliploides, con ventajas sobre los especímenes diploides. De este modo, algunas especies son cultivadas actualmente en regiones en las cuales no crecerían o sobrevivirían los especímenes en sus formas nativas $(3,8,9,22,23)$.

El presente trabajo busca analizar los diferentes métodos de inducción de poliploidías en moluscos bivalvos, para la evaluación de sus ventajas y limitaciones en su aplicación.

\section{INDUCCIÓN DE POLIPLOIDÍAS}

Las poliploidías inducidas en moluscos, si bien implican manipulaciones cromosómicas, no generan en sí, organismos transgénicos, ya que la totalidad del ADN que poseen los animales sometidos a los métodos que se describen en este documento, pertenece a estas mismas especies (genes endógenos) y por definición, se considera a un organismo como transgénico cuando se le ha insertado ADN extraño al de sus genomas (24).

Ahora bien, es necesario comprender las potenciales ventajas que presentan los poliploides sobre los diploides. Inicialmente el desarrollo de poliploides se enfocó en la obtención de triploides, considerando algunas características de éstos, por ejemplo, la mayoría son estériles $(5,6,18,20,25,26,27)$, lo que en principio representa menor riesgo de impacto ambiental al ser introducidos en zonas de las cuales no es endémico, reduciendo su potencial propagación no controlada (28). La misma condición de esterilidad supondría que no invierten recursos metabólicos y energía en desarrollo gonadal 
o reproducción, canalizándolo a su crecimiento somático $(3,6,9,21,25,28)$ y finalmente, presentan mejor calidad de carne, así como mayor heterocigocidad, lo que, eventualmente, les confiere un mayor acervo alélico con el cual enfrentar desafíos patológicos o ambientales $(9,22,25,29,30)$.

Los trabajos pioneros realizados para la obtención de triploides se originaron en la década de los 70, con su inducción en la ostra Crassostrea virginica. Siendo los resultados de Stanley y colaboradores (29), los primeros que reportan triploides viables $(5,6,25,29)$.

Desde entonces, se han generado protocolos de producción para diferentes especies, las cuales han sido utilizadas exitosamente en diferentes regiones.

\section{FUNDAMENTOS DE LA INDUCCIÓN A POLIPLOIDÍAS}

La aplicación de estas técnicas se basa en las características de muchas de sus especies, como almejas, mejillones y pectínidos, en las que los huevos son liberados al medio como ovocitos primarios, sin completar la meiósis $(5,8,25,29,31)$.

Esto significa que los ovocitos realizan las fases de crecimiento G1, síntesis de ADN o fase $\mathrm{S}$ y crecimiento secundario G2, para ser liberados cuando aún presentan un número duplicado de cromosomas, para continuar externamente con el proceso meiótico y finalmente, realizar la singamia $(6,29)$.

Secuencialmente, este proceso implica ovocitos en un estado de meiosis 1"arrestada", si un espermatozoide ingresa, no se une inmediatamente al núcleo, sino que permanece en un pronúcleo independiente, pero activa la continuación del proceso meiótico. El ovocito realiza la primera de dos divisiones celulares continuas, en la primera de ellas libera la mitad del ADN materno presente dentro del primer cuerpo polar (Figura 1) y da lugar a un ovocito secundario "diploide". Se presenta luego otra división celular y liberación del segundo cuerpo polar, para establecer la condición haploide, momento en el cual el contenido del espermatozoide se funde con el núcleo materno, generando el embrión $(5,6,8,20)$.

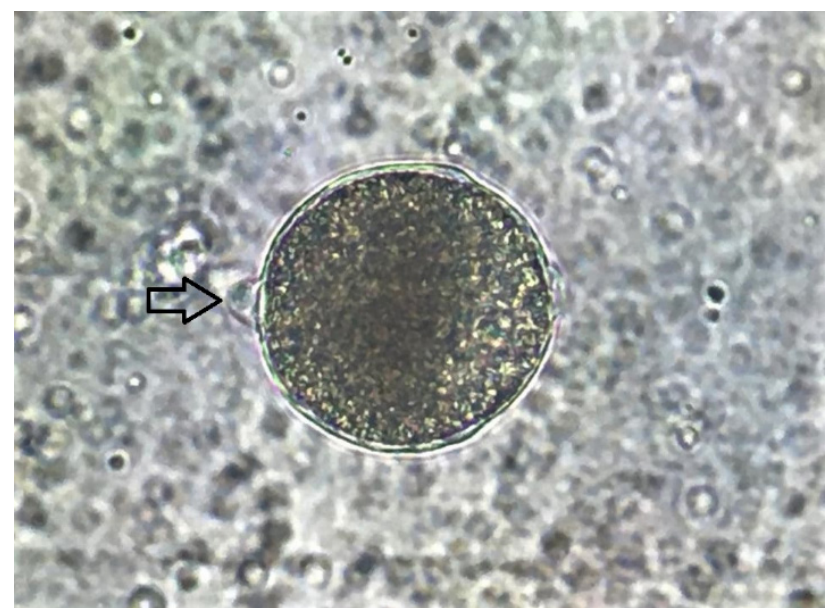

Figura 1. Huevo fecundado de C. gigas. Se observa el primer cuerpo polar (flecha). 400x, sin tinción

Con esta premisa y como se verá a continuación, es posible interferir en el proceso de meiosis y modificar la cantidad final de cromosomas presentes.

\section{TIPOS DE INDUCCIÓN A POLIPLOIDÍAS}

Conceptualmente, la inducción a poliploidías se puede dividir en dos tipos generales, según la naturaleza del proceso utilizado. Categorizando a los métodos en físicos y químicos $(5,6,20,31)$.

Métodos físicos de inducción a poliploidía. En estos métodos, los huevos fecundados se someten a choques de temperatura generalmente altas o a la aplicación de presiones hidrostáticas elevadas con la finalidad de alterar los procesos de división celular $(5,6,31)$. 
Los ciclos de incremento térmico provocan la desnaturalización del huso acromático, necesario para separar los cromosomas durante la anafase, esto impide la segregación y salida de cromosomas en los cuerpos polares. Esta metodología ha sido ensayada en ovocitos fecundados tanto por sometimientos a choques calientes como a alternación de choques fríos y calientes, según la especie de molusco $(8,32,33,34)$.

Por su parte, la aplicación de presión hidrostática impide la salida de los cuerpos polares, debido a la fuerza ejercida sobre el exterior de la célula (35). En este método se someten los ovocitos fecundados a presiones que puede variar de 6000 a 8000 psi, por tiempos específicos $(5,36)$.

Ambas técnicas han presentado diferentes grados de éxito, provocando en ocasiones, resultados irregulares (5, 37), unido al hecho de requerir de los equipos necesarios para los procedimientos.

Adicionalmente, se han ensayado otros métodos, como fusión celular mediante aplicación de pulsos eléctricos. Estos causan rupturas en las células, originando poros en las membranas y pérdida de integridad. Si se aplica a células en contacto estrecho, da lugar a su unión y posterior fusión nuclear, creando así células tetraploides (33).

Métodos químicos de inducción a poliploidía. Requiere de moléculas cuya acción interfiere con alguno de los pasos realizados en la división celular. En estos métodos, se han utilizado diferentes moléculas (Figura 2), como Citocalasina B, 6-Dimetilaminopurina, Cafeína, Nocodazol y Colchicina $(6,8,20,21,23,25,29,31,38,39)$.
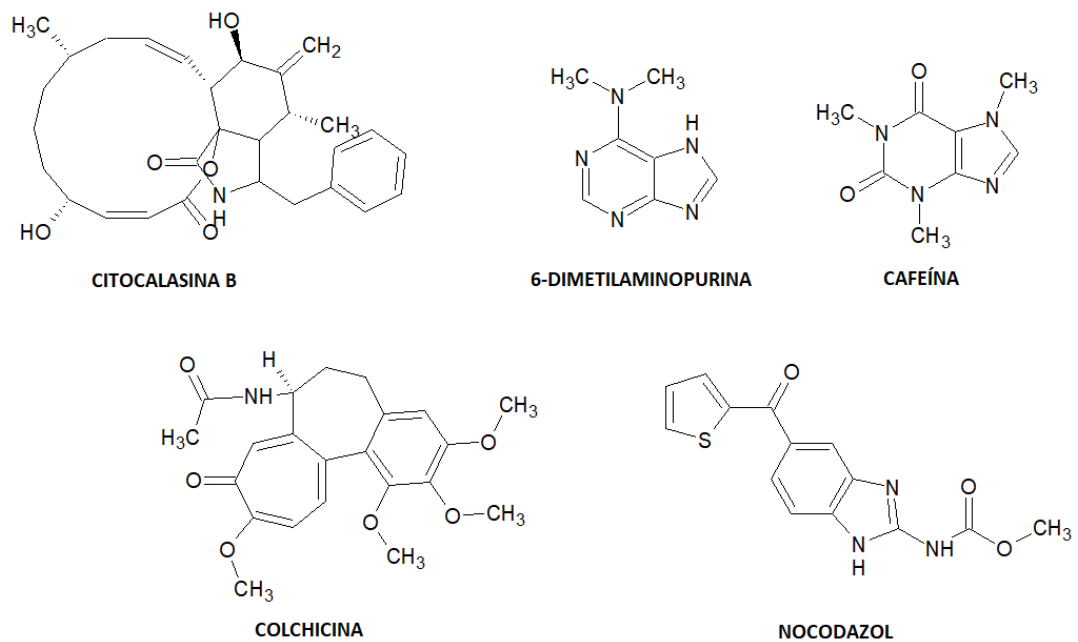

Figura 2. Estructura de algunos inductores de poliploidías

Stanley et al (29), reportaron por primera vez el uso de Citocalasina B, como inductor, aplicándolo durante la meiosis 1 (M1) y dando como resultado embriones poliploides en la ostra Crassostrea virginica (Figura 3). Posteriormente, se obtuvieron resultados en otras especies, como Mytilus edulis y Haliotis discus. Curiosamente, en esta última, la Citocalasina B, no inducía triploidías, sino tetraploidías (25).

Entre los moluscos inducidos químicamente se incluyen Argopecten ventricosus, A. irradians, Crassostrea virginica, C. gigas, Haliotis rufescens, Mytilus edulis, M. galloprovincialis, Mya arenaria, Nodipecten subnodosus, Ostrea edulis, Pinctada fucata, Polymesoda solida, Ruditapes decussatus, Saccostrea commercialis y Spisula solidissima (5,6,20,23,25,29,38,39).

La Citocalasina B es una molécula producida por el hongo Helminthosporeum sp, inicialmente utilizada como antibiótico, su mecanismo de acción propuesto sugiere que obstaculiza la elongación y polimeración del extremo rápido de la actina $(38,40)$, como consecuencia de ello, se imposibilita la citocinesis, ya que se inactiva el anillo extrusor en los ovocitos, formado por microfilamentos de actina y miosina, necesario para la generación de los cuerpos polares, imposibilitando la reducción cromosómica. De este modo, el embrión mantiene un juego extra de cromosomas por cada división no realizada $(5,6,29,35,41)$.

Este efecto de inactivación del anillo de actina fue demostrado en experimentos realizado con la almeja Spisula solidissima, a las cuales se aplicó un inhibidor de la miosina-II ATPasa y dado que la miosina actúa en conjunto con la actina para realizar la contracción, su inhibición impide la extrusión del cuerpo polar (38). 

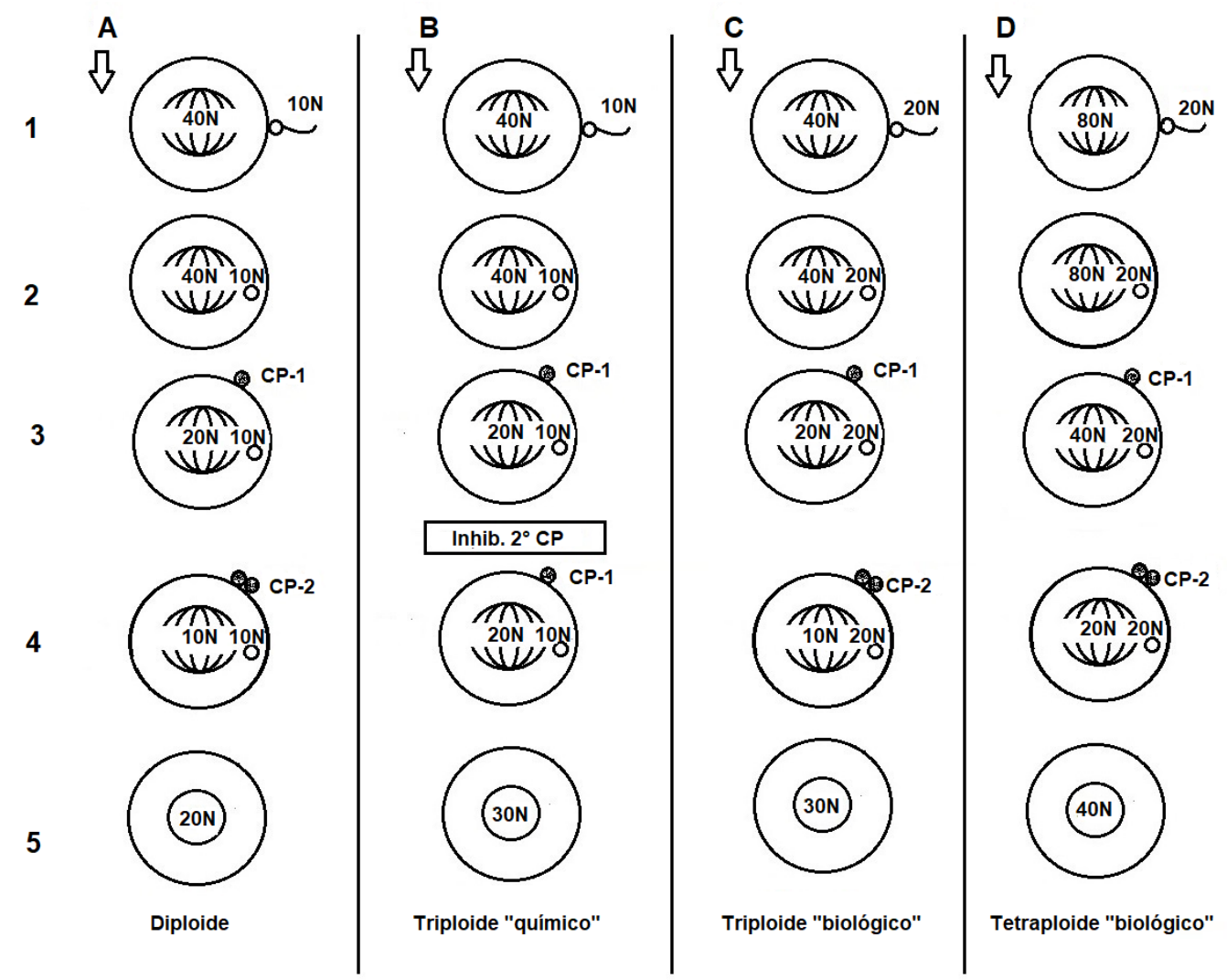

Figura 3. Diagrama de cuatro posibles rutas de desarrollo embrionario en C. gigas (Posterior a fase S). A. Obtención de diploides: 1 Ovocito diploide fertilizado por espermatozoide haploide. 2. Se reactiva la M1. 3. Sale el primer cuerpo polar, primera reducción cromosómica. 4. Sale segundo cuerpo polar, segunda reducción cromosómica. 5. Singamia y formación de embrión diploide. B. Obtención de triploides por inhibición del segundo cuerpo polar: 1 Ovocito diploide fertilizado por un espermatozoide haploide. 2. Se reactiva la M1. 3. Sale el primer cuerpo polar, primera reducción cromosómica. 4. Se inhibe el segundo cuerpo polar, no hay reducción cromosómica. 5. Singamia y formación de embrión triploide. C. Obtención de triploides por cruce de diploide y tetraploide: 1 Ovocito diploide fertilizado por espermatozoide tetraploide. 2. Se reactiva la M1. 3. Sale el primer cuerpo polar, primera reducción cromosómica. 4. Sale el segundo cuerpo polar, segunda reducción cromosómica. 5. Singamia y formación de embrión triploide. D. Obtención de tetraploides por cruce de tetraploides sin inhibición de cuerpos polares: 1 Ovocito tetraploide fertilizado por un espermatozoide tetraploide. 2. Se reactiva la M1. 3. Sale el primer cuerpo polar, primera reducción cromosómica. 4. Sale el segundo cuerpo polar, segunda reducción cromosómica. 5. Singamia y formación de embrión tetraploide.

La Citocalasina B es una sustancia de riesgo para su manejo, generando restricciones (42). Es insoluble en agua, por lo que se usa Dimetilsulfóxido (DMSO) como vehículo y su aplicación requiere sincronía con la formación de los cuerpos polares, así como concentraciones, condiciones y tiempos precisos. Aun así, constituye el método químico más utilizado actualmente $(5,6,8)$.

Maldonado-Amparo et al (5), comentan, además, que el momento y los tiempos de aplicación varían dependiendo de cuál reducción cromosómica se pretende inhibir (M1 o M2), así como también de los tiempos en que cada una de las reducciones cromosómicas ocurren.

Otra molécula utilizada, la 6-Dimetilaminopurina, cuyo mecanismo de acción difiere en el proceso afectado (43), ataca la organización y polimerización de los microtúbulos que forman el huso acromático. Esto imposibilita la segregación cromosómica en el anafase, impidiendo así la reducción cromosómica $(42,44)$ y generando embriones con juegos extra de cromosomas, como ocurre con la Citocalasina B, pero sin sus riesgos asociados (42).

La Cafeína, por su parte, es producto del metabolismo secundario de algunas plantas. Es un derivado purínico que, aplicado durante la maduración del ovocito, actúa sobre la polimerización de microtúbulos, evitando la citocinesis y se ha empleado en la producción de poliploides, por ejemplo, en C. gigas y en Mytilus galloprovincialis $(5,23)$. 
Además de fármacos que afectan la meiosis, se han utilizado otros que actúan sobre los procesos de mitosis en embriones. Como el mitostático Nocodazol, cuya acción consiste en inducir el desensamblaje de los microtúbulos astrales y con ello, la disrupción y desorganización de los filamentos del huso acromático, imposibilitando la segregación cromosómica, su aplicación se debe realizar cuando la mayoría de las células contienen husos metafásicos con aster periféricos, previo al anafase (38).

En el Tabla 1 se resumen características fisicoquímicas de algunas sustancias utilizadas en inducción poliploide, incluyendo como parámetro el logaritmo del coeficiente de reparto octanol/agua (Log P), que permite identificar la afinidad lipídica, y capacidad de atravesar membranas biológicas (45).

Tabla 1. Parámetros fisicoquímicos de inductores de poliploidías

\begin{tabular}{|c|c|c|c|c|c|}
\hline Molécula & Estructura (SMILES*) & $\begin{array}{l}\text { Peso molecular } \\
\left.\text { (g/mol }{ }^{*}\right)\end{array}$ & $\begin{array}{c}\log \mathrm{P} \\
\text { (calculado*) }\end{array}$ & Descripción & Toxicidad $^{* *}$ \\
\hline Citocalasina B & $\begin{array}{c}\mathrm{CC} 1 \mathrm{CCCC}(\mathrm{C}=\mathrm{CC}(=0) \\
\text { OC23C }(\mathrm{C}=\mathrm{CC} 1) \\
\mathrm{C}(\mathrm{C}(=\mathrm{C}) \mathrm{C}(\mathrm{C} 2 \mathrm{C}(\mathrm{NC} 3=0) \\
\mathrm{CC} 4=\mathrm{CC}=\mathrm{CC}=\mathrm{C} 4) \mathrm{C}) 0) 0\end{array}$ & 479,61 & 3,4 & $\begin{array}{l}\text { Inhibidor de los filamentos de } \\
\text { actina. Muy utilizada en estudios } \\
\text { de transportadores de glucosa y en } \\
\text { ensayos de micronúcleos in vitro. }\end{array}$ & $\begin{array}{l}\text { La prueba de letalidad } \\
\text { en Artemia salina es: } \mathrm{LC}_{50} \\
22,8 \mu \mathrm{g} / \mathrm{ml} \\
\mathrm{LD}_{50} \text { en ratas: } 11 \mathrm{mg} / \mathrm{kg}\end{array}$ \\
\hline 6-Dimetilaminopurina & $\mathrm{CN}(\mathrm{C}) \mathrm{c} 1 \mathrm{ncnc} 2 \mathrm{nc}[\mathrm{nH}] \mathrm{c} 12$ & 163,18 & 4,1 & $\begin{array}{l}\text { Inhibidor de quinasas y quinasas } \\
\text { ciclo-dependientes, de uso en } \\
\text { patogénesis y estudios de meiosis }\end{array}$ & ----------- \\
\hline Cafeína & $\begin{array}{c}\mathrm{Cn} 1 \mathrm{cnc} 2 \mathrm{c} 1 \mathrm{c}(=0) \mathrm{n}(\mathrm{C}) \\
\mathrm{c}(=0) \mathrm{n} 2 \mathrm{C}\end{array}$ & 194,19 & 0,06 & $\begin{array}{l}\text { Metilxantina, con acción antagonista } \\
\text { en receptores de adenina e inhibidor } \\
\text { de la fosfodiesterasa de cAMP. }\end{array}$ & $\begin{array}{l}\text { En humanos existe la } \\
\text { controversia sobre si } \\
\text { su uso en altas dosis } \\
\text { (>200mg/día) se } \\
\text { relaciona a daño hepático }\end{array}$ \\
\hline Nocodazol & $\begin{array}{l}\operatorname{COC}(=0) N c 3 n c 2 \operatorname{cc}(\mathrm{C}(=0) \\
\mathrm{c} 1 \mathrm{cccs} 1) \operatorname{ccc} 2[\mathrm{nH}] 3\end{array}$ & 301,32 & 2,79 & $\begin{array}{c}\text { Antimitótico, altera los } \\
\text { microtúbulos, por unión a la } \\
\beta \text {-tubulina. Detiene el ciclo celular } \\
\text { en G2/M. Se ha utilizado en edición } \\
\text { genómica CRISPR }\end{array}$ & $\begin{array}{c}\text { La LD } \\
30 \text { en ratones es: } \\
39,53 \mathrm{mg} / \mathrm{kg}\end{array}$ \\
\hline Colchicina & $\begin{array}{c}\mathrm{COc} 3 \mathrm{cc} 2 \mathrm{CC}[\mathrm{C} @ \mathrm{H}] \\
(\mathrm{NC}(\mathrm{C})=0) \mathrm{c} 1 \mathrm{cc}(=\mathrm{O}) \mathrm{c}(\mathrm{OC}) \\
\mathrm{ccc} 1 \mathrm{c} 2 \mathrm{c}(\mathrm{OC}) \mathrm{c} 30 \mathrm{C}\end{array}$ & 399,44 & 1,1 & $\begin{array}{l}\text { Inhibidor de división celular, } \\
\text { en ensayos de intercambio de } \\
\text { cromátidas hermanas, estudios } \\
\text { de actividad antimicrobiana y } \\
\text { citotóxica, y en la disrupción de } \\
\text { microtúbulos }\end{array}$ & $\begin{array}{l}\text { En altas dosis es } \\
\text { hepatotóxica en humanos }\end{array}$ \\
\hline
\end{tabular}

*https://www.molinspiration.com/; **https://pubchem.ncbi.nlm.nih.gov/

Fuente: 46; $47 ; 48 ; 49$

\section{PROBLEMAS DE LA INDUCCIÓN DE POLIPLOIDÍA QUÍMICA}

Estos métodos poseen limitantes importantes, entre ellas: requieren del uso de sustancias peligrosas o restringidas, los resultados de triploidía no alcanzan el 100\% de los embriones tratados, las sobrevivencias larvales son generalmente bajas, y en muchas ocasiones se presentan reversiones cromosómicas durante el desarrollo $(3,5,50)$.

Adicionalmente, pueden generarse tasas considerables de irregularidades cromosómicas, incluyendo presencia de organismos aneuploides y heteroploides o "mosaicos" $(28,30)$.

Como solución a estos problemas y con la finalidad de obtener una producción suficiente de triploides, se planteó como estrategia obtenerlos mediante el cruce de tetraploides y diploides $(3,21,51)$, dando como resultado a los llamados "triploides biológicos" (5,20) (Figura 3).

Evidentemente, para este método, la disponibilidad de reproductoras tetraploides es fundamental $(1,5,52)$. 


\section{PRODUCCIÓN DE TETRAPLOIDES}

Su consecución significó un desafío debido a múltiples obstáculos técnicos, tales como la presencia de una baja tasa de generación de organismos viables (22), anomalías cromosómicas obtenidas por métodos químicos (28), baja fertilidad de los organismos utilizados para su obtención o baja sobrevivencia de los organismos obtenidos, los cuales, en la mayoría de los casos, no superaban los estadíos larvales $(5,30)$.

Teóricamente, los tetraploides pueden ser obtenidos por diferentes métodos, como inhibición de la meiósis en diploides, cruces de diploides y triploides, inhibición de la mitosis en huevos fecundados con mitostáticos, fusión celular o mediante ginogénesis, utilizando esperma inactivado mediante radiación UV combinada con inhibición química de los cuerpos polares $(6,8,21,25,29,33,38,39,53)$.

Uno de los métodos de mayor aplicación es el uso de ovocitos de triploides, fecundados con espermas diploides, e inhibición del primer cuerpo polar, pero permitiendo la liberación del segundo (22,26,51), sin embargo, el proceso no es simple, ya que se han observado bajas sobrevivencias en estadíos larvales, las anomalías cromosómicas ya mencionadas, bajas tasas de tetraploides adultos con capacidad reproductiva e instabilidad cromosómica $(5,27,30)$.

Sin embargo, una vez obtenidos tetraploides adultos, es posible obtener más individuos mediante el cruce de progenitores tetraploides, sin inhibir cuerpos polares (Figura 3), ya que ambos gametos presentan un contenido cromosómico duplicado (22).

\section{DETERMINACIÓN DE PLOIDIAS}

Posterior a la obtención de los poliploides, es necesario disponer de metodologías que permitan establecer esta condición.

A pesar de que frecuentemente los tetraploides presentan morfologías irregulares, esto es insuficiente para establecer su carga genética. Ya que tanto diploides como poliploides pueden presentar irregularidades fenotípicas, siendo necesario establecer las ploidías a nivel genotípico.

Para ello, se utilizan procedimientos que revelan la carga cromosómica. Una de estas técnicas es el análisis cariológico, el cual se fundamenta en la fijación de muestras, tinción, observación microscópica y conteo de los cromosomas presentes $(3,23,29,30,31)$. Variaciones de esta técnica han utilizado el apoyo de programas de análisis de imágenes (22).

Otra metodología, la citometría de flujo, se fundamenta en el análisis de las características ópticas y emisión de fluorescencia de las muestras procesadas (3,26,30,31,36,37) (Figura 4). Para el análisis de ploidías, se tiñen células libres con Ioduro de propidio (PI) u otra sustancia análoga, que se adhiere al ADN. Posteriormente las muestras son irradiadas con láser y analizadas durante su paso por capilares, donde sus espectros son captados y procesados por el equipo. A mayor cantidad de PI por partícula analizada, mayor fluorescencia y mayor contenido de ADN, permitiendo determinar los diferentes grados de ploidía en las muestras, las cuales son mostradas como curvas cuyos picos indican las proporciones relativas de ADN (54).
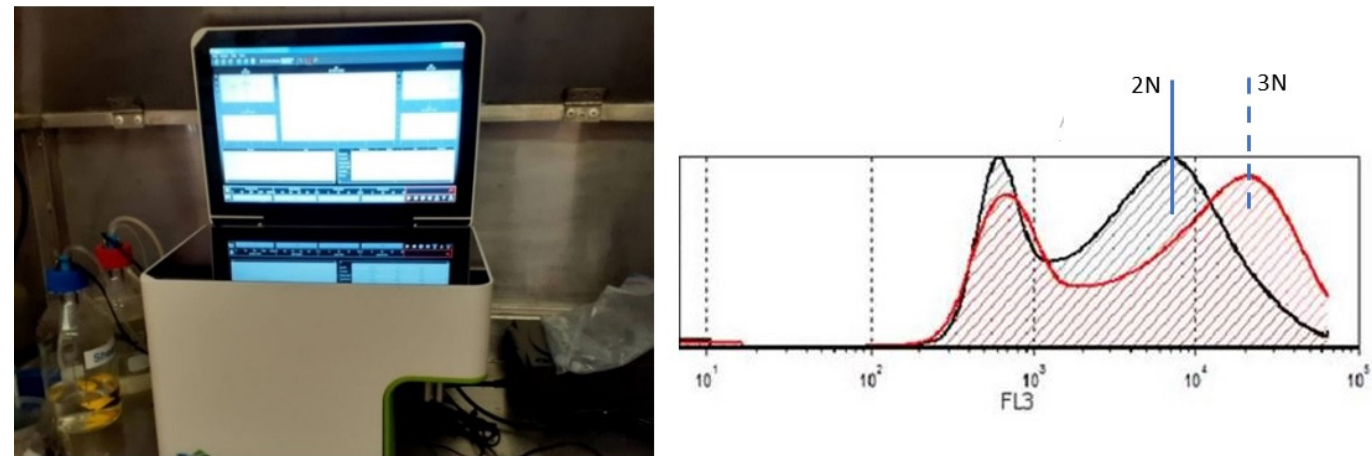

Figura 4. Izquierda: Citómetro de flujo, utilizado en la determinación del contenido de ADN relativo en las células analizadas. Derecha: curva logarítmica obtenida de dos muestras, en este caso una diploide $(2 \mathrm{~N})$ y una triploide $(3 \mathrm{~N})$. 


\section{DISCUSIÓN}

Tal como se mencionó, la producción de poliploides, en especial de triploides, presenta ventajas sobre los diploides, lo que los convierte en un producto altamente aceptado por los productores, siendo su principal rasgo el escaso desarrollo gonádico. Causando esterilidad parcial o total $(18,21,25,26,27)$. La cual se atribuye a una sinapsis irregular cuando tres juegos de cromosomas homólogos coinciden en un núcleo durante la profase de la M1 (5,20,30).

Todos los métodos de inducción presentan limitantes, los métodos físicos como temperaturas y presiones, además de requerir equipos y condiciones especiales, no garantizan la consecución de poliploides estables u homogéneos. Adicionalmente, los resultados pueden diferir fuertemente entre tratamientos y entre especies, pese a ello, se han logrado generar poliploidías en muchos ensayos $(6,8,20,32,34)$, por lo que se deben considerar las particularidades de cada especie, así como las condiciones y metodologías aplicadas.

Esta irregularidad de resultados podría deberse a las condiciones y tiempos de aplicación de los tratamientos. Debe existir una certeza total sobre el momento adecuado de aplicación del proceso, requiriendo para ello de sincronía en los ovocitos presentes, de modo que la mayoría presente la misma fase de meiósis, así como su identificación certera (5,6,32).

Este mismo obstáculo se presentaría en las inducciones químicas, ya sea por el uso de inhibidores de meiosis o de mitosis $(6,8,20,21,25,29,38,39)$.

Paralelo al momento de aplicación, deben considerarse aspectos, como dosis utilizada, que, en el caso de la Citocalasina $\mathrm{B}$, ha sido utilizada con frecuencia en concentraciones de 0.5 a $1 \mathrm{ppm}$, requiriendo del DMSO en la misma concentración $(21,33,37)$. Del mismo modo, es relevante el tiempo de exposición al fármaco, que puede variar entre especies e incluso entre condiciones de uso. El retiro del fármaco debe ser asimismo rápido y eficiente para garantizar la interrupción de su acción, pero sin lesionar los embriones $(5,8,29,37,38)$.

Sobre el control de los tiempos, generalmente se utiliza un grupo control en paralelo, formado por muestras no tratadas con el fármaco. Estas muestras se mantienen en las mismas condiciones y son monitoreadas para establecer el momento de formación de los cuerpos polares o divisiones celulares, usando esta información para determinar los tiempos de aplicación o retiro del inductor sobre el grupo a tratar (37).

Aun con estas variables controladas, los resultados no son siempre los esperados y pueden presentarse bajas sobrevivencias o irregularidades en el contenido genético del producto, incluyendo aneuploidías y heteroploidías, lo que suele traducirse en altas mortalidades en estadíos larvales y en falta de homogeneidad, con poca o nula sobrevivencia post metamorfosis (8). A ello se le debe sumar el tema de la peligrosidad en el caso de la Citocalasina B $(5,6)$.

Ahora bien, a pesar de que las metodologías utilizadas para la producción de poliploides generan aneuploides y heteroploides en diferentes proporciones, su existencia no es exclusiva de animales tratados con inductores. La pérdida espontanea de cromosomas en tejidos somáticos de $C$. gigas ha sido reportada en muestras naturales y aparentemente sanas, así como en especímenes afectados por algunos tipos de neoplasias. Pero en este caso, serían el resultado de procesos patológicos indeseados $(19,38,55)$, sin ventajas zootécnicas.

Sobre que cuerpo polar se debe inhibir, dependerá del tipo de proceso y especie tratada. Se han realizado trabajos basados en la inhibición del primer cuerpo polar. Por ejemplo, Guo y Allen (56), lograron producir tetraploides de $C$, gigas a partir de huevos triploides, fecundados con esperma haploide usando Citocalasina B. La aplicación de Cafeína en Polymesoda solida brindó resultados interesantes, pero también generó aneuploidías (23). Por su parte, Hernández et al (20), produjeron tetraploides en Haliotis rufescens, mediante ginogénesis combinada con inhibición del primer cuerpo polar, pero el producto no logró superar la fase de trocófora.

Del mismo modo, se han realizado trabajos sobre la inhibición del segundo cuerpo polar, por ejemplo, McCombie y et al (22), aplicaron Citocalasina B en $C$, gigas, para obtener tetraploides. Adicionalmente, se han aplicado metodologías de inhibición de ambos cuerpos polares, usando esperma previamente inactivado para reanudar la meiosis $(33,38,57,58)$. Aunque se han logrado generar tetraploides por estos métodos, las sobrevivencias obtenidas posterior a la metamorfosis han sido, en general, bajas (6).

Como solución a algunos de los problemas de las inducciones físicas y químicas, se optó por la obtención de tetraploides, recurriendo a estrategias como inhibición de meiosis, mitosis y ginogénesis, o combinación de ellas, para su uso como reproductores y obtener triploides biológicos $(26,29,33)$. 
Las primeras obtenciones de tetraploides se basaron, nuevamente, en la inhibición química, esta vez del primer cuerpo polar en huevos de diploides fertilizados con esperma haploide. En estos casos, los tetraploides obtenidos se suponen consecuencia de un evento aleatorio, posiblemente asociado a discrepancias en la segregación cromosómica en arreglos tri y unipolares, en donde un juego completo de cromosomas es liberado en el segundo cuerpo polar, con tres juegos retenidos (57). En estos casos, ocasionalmente también se generan pentaploides, como consecuencia de una inhibición indeseada de ambos cuerpos polares (37).

Por lo anterior, el uso de diploides para generar tetraploides, presentó obstáculos, que aun en el caso de utilizar protocolos correctos, no garantizaban los resultados, con una muy baja sobrevivencia de los productos obtenidos. Este efecto se podría explicar en parte debido al limitado tamaño de los ovocitos de los diploides, en los cuales se dificultaba la adecuada contención de juegos duplicados del contenido cromosómico característico de los tetraploides $(5,22)$.

Como solución, se recurrió al uso de triploides, como en C. gigas, cuya fertilidad es muy baja pero no nula (26,50). Los ovocitos triploides presentan un diámetro aproximadamente $20 \%$ mayor que en los diploides (26), lo que les permitiría teóricamente, albergar mejor los cromosomas adicionales implícitos en las poliploidías $(5,29)$.

Esto permitió la obtención de reproductores tetraploides viables, pero, con baja sobrevivencia. Posiblemente debido a que las rutas de la sinapsis cromosómica pueden variar considerablemente entre individuos triploides, presentando sinapsis univalentes, bivalentes y trivalentes. Estas variaciones pueden influir en eventos meióticos posteriores, afectando la viabilidad de los embriones durante su desarrollo (27).

El producto final obtenido sería entonces determinado por las segregaciones cromosómicas realizadas. Donde la segregación bipolar es la única que da lugar a tetraploides viables. Las altas mortalidades presentes en larvas de los desoves destinados a generar tetraploides serían una consecuencia normal e ineludible de la técnica en sí y de la forma en que se segregan los cromosomas $(5,26,27,30)$.

Otras estrategias utilizadas, como la ginogénesis, tratando a los espermatozoides con radiación UV, inactivando la funcionalidad de su carga genética y aplicando inductores para impedir la liberación de cuerpos polares. Sobre esta técnica, el principal punto de control lo representa el tratamiento UV per se, la intensidad y tiempo de irradiación deben ser minuciosamente controlados, así como las concentraciones de los espermatozoides utilizados y el espesor de la capa donde se contienen. Tratamientos inadecuados, por defecto o por exceso, pueden implicar la no total inactivación de los cromosomas masculinos o daños sobre el flagelo, imposibilitando en ambos casos, la consecución de tetraploides (20).

Por su parte, el método de fusión celular resulta muy sensible a la orientación del embrión con respecto al campo eléctrico utilizado, brindando resultados irregulares (33). En tanto que las inhibiciones de la mitosis, tampoco han brindado resultados completamente satisfactorios, dando muy baja o ninguna sobrevivencia $(6,33,39,59)$.

Pese a ello, la metodología a seguir, una vez obtenidos los tetraploides es, teóricamente más fácil, cruzando adultos tetraploides para obtener progenie tetraploide. Inesperadamente, en ocasiones estos cruces no generan tetraploides. McCombie y colaboradores (22), argumentan que esto podría deberse a un número desequilibrado de cromosomas en los ovocitos o a una pérdida somática de cromosomas. Aun así, generalmente se ha logrado generar tetraploides por diferentes métodos.

Con esta panorámica, las investigaciones continuarán. Los procedimientos físicos y químicos, pese a su baja tasa de éxito relativo y efectos colaterales, seguirán siendo realizados para producir los tetraploides, necesarios para generar los triploides demandados por el mercado.

Los fármacos usados, sus mecanismos de acción, dosis, modo y tiempos de aplicación, seguirán siendo sometidos a optimizaciones, sin descartar estrategias como la ginogénesis.

Finalmente, como ya se mencionó, los poliploides generados por los métodos no insertan ADN exógeno, por lo que no califican como organismos transgénicos, sino cromosómicamente manipulados, con importantes aplicaciones zootécnicas.

\section{Conflictos de interes}

Declaramos no tener conflictos de interés en el trabajo presentado 


\section{REFERENCIAS}

1. FAO. El estado mundial de la pesca y la acuicultura. Cumplir los objetivos de desarrollo sostenible. Roma: Food and Agriculture Organization of the United Nations; 2018; http://www.fao.org/3/i9540es/i9540es.pdf

2. Lodeiros C, Rodríguez-Pesantes D, Márquez A, Revilla J, Chávez-Villalba J, Sonnenholzner S. Suspended cultivation of the Pacific oyster Crassostrea gigas in the Eastern Tropical Pacific. Aquacult Int. 2017; 26:337-347. https://doi. org/10.1007/s10499-017-0217-z

3. Nell J A. Farming triploid oysters. Aquaculture. 2002; 210:69-88. http://pacifichybreed.com/wp-content/ uploads/2019/11/Nell-2002-Aquaculture-farming-triploid-oysters.pdf

4. Cáceres J, Vásquez R. Manual de buenas prácticas para el cultivo de moluscos bivalvos. OIRSA OSPESCA. 2014. https://isamx.org/sitio/pdfs/Manual\%20de_BPde_M_Version\%20Digital 011014155613.pdf

5. Maldonado-Amparo R, Ruiz C A, Ibarra A M, Rueda-Puente E O, del Toro C L, Rodríguez F. Poliploidía en moluscos de importancia comercial. A Review. European Scientific Journal. 2016; 12(33):1857-7881. https://doi.org/10.19044/ esj.2016.v12n33p69

6. Yang H, Guo X, Scarpa J. Induction and Establishment of Tetraploid Oyster Breeding Stocks for Triploid Oyster Production. FA215. School of Forest Resources and Conservation, Program in Fisheries and Aquatic Sciences, UF/ IFAS Extension. 2019; https://edis.ifas.ufl.edu/fa215

7. Varela A, Barbosa W. Captura, selección e ingestión de partículas en Ostreidae (Bivalvia): Crassostrea spp. AquaTechnica, 2020; 2(3):161-181. http://dx.doi.org/10.33936/at.v2i3.3091

8. Guo X, Wang Y, Xu Z, Yang H. Chromosome Set Manipulation in Shellfish. In: New Technologies in Aquaculture: Improving Production Efficiency, Quality and Environmental Management (eds. Burnell, G \& Allen, G), Woodhead Publishing Limited: Abington, Cambridge; 2009

9. Villanueva-Fonseca B P, Góngora-Gómez A M, Muñoz-Sevilla N P, Domínguez-Orozco A L, Hernández-Sepúlveda J A, García-Ulloa M, Ponce-Palafox J T. Growth and economic performance of diploid and triploid Pacific oysters Crassostrea gigas cultivated in three lagoons of the Gulf of California. Lat. Am. J. Aquat. Res., 2017; 45(2):466-480. http://dx.doi.org/10.3856/vol45-issue2-fulltext-21

10. Mazón S J M. Cultivo del Ostión Japonés Crassostrea gigas. En: Casas, V.; Ponce, D, G. eds. Estudio del potencial pesquero y acuícola de Baja California Sur. Baja California Sur, México: 1996. http://dspace.cibnor.mx:8080/ handle/123456789/1723

11. Rodríguez-Quiroz G, García-Ulloa G, Domínguez-Orozco A L, Valenzuela-Hernández T N, Nava-Pérez E, GóngoraGómez A M. Relación del crecimiento, condición y supervivencia del ostión del Pacífico Crassostrea gigas y las variables ambientales, cultivado en suspensión en el Sistema lagunar Navachiste-Macapule, Sinaloa, México. Revista de Biología Marina y Oceanografía. 2016; 51(3):541-551. http://dx.doi.org/10.4067/S0718-19572016000300006

12. Escudeiro A. Crecimiento y reproducción de la ostra rizada, Crassostrea gigas (Thunberg, 1793), cultivada en intermareal y en Batea de Galicia (NW España). Universidade do Algarve Facultade de ciencias do mar e do ambiente. Centro de investigacóns mariñas (CIMA). Consellería de Pesca, Marisqueo e Acuicultura. Xunta de Galicia. 2006. https://core.ac.uk/download/pdf/61504226.pdf

13. García-Ulloa M, Góngora-Gómez A M, Hernández-Sepúlveda J A. Manual para el policultivo de bivalvos en México. Validación tecnológica. Aqua Ediciones. 2018.

14. Chavez-Villalba J, Pommier J, Andriamiseza J, Pouvreau S, Barret J, Cochard J C, Le Pennec M. Broodstock conditioning of the oyster Crassostrea gigas: origin and temperature effect. Aquaculture. 2002; (214):115-130. http://dx.doi. org/10.1016/S0044-8486(01)00898-5

15. García-Bernal M, Medina-Marrero R, Mazón-Suástegui J M, Arcos-Ortega G F, Tordecillas-Guillén J L, Barajas-Ponce U. Preengorda de semillas de ostra americana Crassostrea virginica con aplicación de actinomicetos probióticos y medicamentos homeopáticos. AquaTechnica. 2020; 2(3):150-160. http://dx.doi.org/10.33936/at.v2i3.3086 
16. Martínez P. Aplicaciones de la genética para la mejora de la acuicultura. Bol. Inst. Esp. Oceanogr. 2005; 21(1-4):225238. https://core.ac.uk/download/pdf/71764913.pdf

17. Rodriguez-Romero F, Gasca-Montes de Oca M. Los cromosomas del hibrido experimental de Crassostrea Virginica Gmelin 1791 y Crassostrea rhizophorae Guilding 1828 (Pseudolamellibranchiata: ostreidae). Ciencias Marinas. 1998; 24(1):55-63. http://dx.doi.org/107773/cm.v24l.736

18. Que H, Allen SK. Hybridization of tetraploid and diploid Crassostrea gigas (Thumberg) with diploid C. ariakensis (Fujita). Journal of shellfish Research. 2002; 27:137-143. https://core.ac.uk/download/pdf/235399191.pdf

19. Thiriot-Quievreux C. Review of literature of bivalve cytogenetic in the last ten years. Cahiers de Biologie Marine. 2002; (43) 17-26. http://dx.doi.org/10.21411/CBM.A.4BE6D552

20. Hernández NK, Marquéz C, Cruz P. Poliploidía y ginogénesis en abulón rojo (Haliotis rufescens), certificación genética de genética de abulón rojo y azul (Haliotis fulgens), y cariotipos de especies parentales. [Tesis de maestría]. Centro de Investigaciones Biológicas del Noroeste; México; 2004.

21. Allen SK. Triploid oysters ensure year-round supply. Oceanus. 1988; 31:58-63.

22. McCombie H, Ledu C, Phelipot $\mathrm{P}$, Lapègue $\mathrm{S}$, Boudry $\mathrm{P}$, Gérard A. A complementary method for production of tetraploid Crassostrea gigas using crosses between diploids and tetraploids with Cytochalasin B treatments. Marine Biotechnology. 2005; 7(4):318-330. http://dx.doi.org/10.1007/s10126-004-0440-2

23. Revilla D, Báez F, García Y, Severeyn H, Villamediana P. Inducción química de poliploidía en el molusco bivalvo Polymesoda solida (Philippi, 1846) (Bivalvia: Corbiculidae). Boletín del Centro de Investigaciones Biológicas. 2016; 50(2):121-133. https://produccioncientificaluz.org/index.php/boletin/article/view/22901

24. Rasmussen R S, Morrissey M T. Biotechnology in Aquaculture: Transgenics and Polyploidy. Comprehensive Food Science and Food Safety. 2007; 6(1):2-16. https://doi.org/10.1111/j.1541-4337.2007.00013.x

25. Guo X, Cooper K, Hershberger W K, Chew K K. Genetic Consequences of Blocking Polar Body I with Cytochalasin B in Fertilized Eggs of the Pacific Oyster, Crassostrea gigas: I. Ploidy of Resultant Embryos. Biol Bull. 1992; 183:381-386. https://doi.org/10.2307/1542013

26. FAO. Food and Agriculture Organization of the United Nations. (2006). Cultivo de bivalvos en criadero Un manual práctico. Documento técnico de pesca 471. Roma, Italia; 2006. http://www.fao.org/3/y5720s/y5720s00.htm

27. Que H, Guo X, Zhang F, Allen S K. Chromosome segregation in fertilized eggs from triploid Pacific oysters, Crassostrea gigas (Thunberg), following inhibition of polar body 1. Biological Bulletin, 1997; 193(1):14-19. https://doi. org/10.2307/1542732

28. Wang Z, Guo X, Allen S K, Wang R. Aneuploid Pacific oyster (Crassostrea gigas, Thunberg) as incidentals from triploid production. Aquaculture 1999; 173:347-357. https://doi.org/10.1016/S0044-8486(98)00457-8

29. Stanley J G, Allen S K, Hidu H. Polyploidy induced in the American oyster, Crassostrea virginica, with Cytochalasin B. Aquaculture, 1981; 23:1-10. https://doi.org/10.1016/0044-8486(81)90002-8

30. de Sousa JT, Allen SK, Wolfe BM, Moss JA. Mitotic instability in triploid and tetraploid one-year-old eastern oyster, Crassostrea virginica, assessed by cytogenetic and flow cytometry techniques. Genome. 2018; 61(2). https://doi. org/10.1139/gen-2017-0173

31. Piferrer F, Beaumont A, Falguière J C, Flajšhans M, Haffray P, Colombo L. Polyploid fish and shellfish: Production, biology and applications to aquaculture for performance improvement and genetic containment. Aquaculture. 2009; 293:125-156. https://doi.org/10.1016/j.aquaculture.2009.04.036

32. Yamamoto S, Sugawara Y. Induced triploidy in the mussel Mytilus edulis, by temperature shock. Aquaculture. 1988; 72:21-29 https://doi.org/10.1016/0044-8486(88)90143-3 
33. Li X. Experimental production of tetraploid oysters for use as broodstock for commercial hatchery production of triploids. FRDC Project No. 1994/081. SARDI Aquatic Sciences Publication: Australia. 2007; https://www.frdc.com. au/Archived-Reports/FRDC\%20Projects/1994-081-DLD.PDF

34. Canello OF, Paredes OL, Toro YJ. Inducción a la triploidía en el ostión del norte Argopecten purpuratus, por medio de shock térmico de calor. Inv Pesq Chile. 1992; 37:5-11.

35. Downing SL; Allen S K. Induced Triploidy in the Pacific Oyster, Crassostreagigas: optimum treatments with Cytochalasin B Depend on Temperature. Aquaculture. 1987; 61:1-15. https://doi.org/10.1016/0044-8486(87)90332-2

36. Chaiton J, Allen S K. Early detection of triploidy in the larvae of Pacific oysters, Crassostrea gigas, by flow cytometry. Aquaculture. 1985; 48:35-43. https://doi.org/10.1016/0044-8486(85)90050-X

37. Maldonado-Amparo R, Ibarra, A M, Ramírez J L. Inducción a la tetraploidía en almeja catarina, Argopecten ventricosus (Sowerby II, 1842). Ciencias Marinas. 2003; 29(2):229-238. http://dx.doi.org/10.7773/cm.v29i2.143

38. Pielak R M, Gaysinskayay V A, Cohen W D. Formation and function of the polar body contractile ring in Spisula. Developmental Biology. 2004; 269:421-443. https://doi.org/10.1016/j.ydbio.2004.01.033

39. Yang H, Guo X. Tetraploid induction by inhibiting mitosis I with heat shock, cold shock, and Nocodazole in the hard clam Mercenaria mercenaria (Linnaeus, 1758). Mar Biotechnol. 2006; 8:501-510. https://doi.org/10.1007/ s10126-005-6183-x

40. MacLean-Fletcher S, Pollard T D. Mechanism of Action of Cytochalasin B on Actin Cell. 1980; 20:329-341. https:// doi.org/10.1016/0092-8674(80)90619-4

41. Zalacain M, Sierrasesúmaga L, Patiño A. El ensayo de micronúcleos como medida de inestabilidad genética inducida por agentes genotóxicos. An Sit Sanit Navar. 2005; 28(2):227-236. https://hdl.handle.net/10171/23747

42. Peachey B L, Allen S K. Evaluation of cytochalasin B and 6-dimethylaminopurine for tetraploidy induction in the Eastern oyster, Crassostrea virginica. Aquaculture. 2016; 450:199-205. https://doi.org/10.1016/j.aquaculture.2015.07.034

43. Tian C, Wang R, Liang Y, Wang Z, Yu R. Triploidy of Crassostrea gigas induced with 6-DMAP: by blocking of the second polar body of the zygotes. J Fish Sci. China 1999; 6(2):1-4. https://www.sciengine.com/publisher/zhongkeqikan/ journal/JFSC/27/7?slug=browse

44. Desrosiers R R, Gerard A, Peignon J M, Naciri Y, Dufresne L, Morasse J, Ledu C, Phelipot P, Guerrier P, Dube F. A novel method to produce triploid embryos in bivalve mollusks by the use of 6-dimethylaminupurine. J Exp Mar Biol Ecol. 1993; 170:29-43. https://doi.org/10.1016/0022-0981(93)90127-A

45. Saha S, Pal D. Log P. Encyclopedia of Physical Organic Chemistry (1ํaed.). USA: Zerong Wang; 2017.

46. Dufresne L, Néant I, ST-Pierre J, Dubé F, Guerrier P. Effects of 6-dimethylaminopurine on microtubules and putative intermediate filaments in sea urchin embryos, J Cell Science. 1991; 99:721-730. https://journals.biologists.com/jcs

47. Ghosh S, Jha S. Colchicine - an Overview for Plant Biotechnologists. In: Ramawat K., Merillon J. (eds) Bioactive Molecules and Medicinal Plants. Springer: Berlin, Heidelberg; 2008; https://doi.org/10.1007/978-3-540-746034 11

48. Feitosa A, Dias A, Ramos G, Bitencourt H, Siqueira J, Marinho P. Lethality of cytochalasin B and other compounds isolated from fungus Aspergillus sp. (Trichocomaceae) endophyte of Bauhinia guianensis (Fabaceae). Rev Argent Microbiol. 2016; 48(3):259-263. https://doi.org/10.1016/j.ram.2016.04.002

49. Pisciottani A, Biancolillo L, Ferrara M, Valente D, Sardina F, Monteonofrio L, Camerini S, Crescenzi M, Soddu S, Rinaldo S. HIPK2 Phosphorylates the Microtubule-Severing Enzyme Spastin at S268 for Abscission. Cells. 2019; 8(7) 684. https://doi.org/10.3390/cells8070684

50. Allen S K. Estimating the ecological impact of triploid Crassostrea gigas: what is the meaning of sterility? FASIP Leave Report, Unité de Recherche en Génétique et Encloserie, IFREMER. La Tremblade: France; 1994. 
51. Guo X, de Brosse G A, Allen S K. All triploid Pacific oysters (Crassostrea gigas Thunberg) produced by mating tetraploids and diploids. Aquaculture. 1996; 142:149-161. https://doi.org/10.1016/0044-8486(95)01243-5

52. Yang H, Guo X, Scarpa J. Tetraploid Induction and Establishment of Breeding Stocks for All-Triploid Seed Production: FA215, 5/2019. UF/IFAS. 2019; 2019:3. https://doi.org/10.32473/edis-fa215-2019

53. Qui L, Kijima A. Gynogenetic tetraploid larvae of the pacific oyster Crassostrea gigas induced by inhibition of the 1st and 2nd meiotic divisions. Tohoku J of Agric Res. 2006; 57(1-2):1-10. http://hdl.handle.net/10097/40396

54. Adan A, Alizada G, Kiraz Y, Baran Y, Nalbant A. Flow cytometry: basic principles and applications, Critical Reviews in Biotechnology, 2016; https://doi.org/10.3109/07388551.2015.1128876

55. Figueras A, Novoa B. Enfermedades de moluscos bivalvos de interés en Acuicultura. Fundación Observatorio Español de Acuicultura - Consejo Superior de Investigaciones Científicas- Ministerio de Medio Ambiente y Medio Rural y Marino: España; 2011.

56. Guo X, Allen S K. Viable tetraploids in the pacific Oyster (Crassostrea gigas Thunberg) produced by inhibition polar body $\mathrm{l}$ in eggs from triploids. Molecular Marine Biology and Biotechnology, 1994; 3(1):42-50. http://www. springerlink.com/openurl.asp?genre=journal\&issn=1053-6426

57. Scarpa J, Wada K T, Komaru A. Induction of tetraploidy in mussels by suppression of polar body formation. Nippon Suisan Gakkaishi. 1993; 59:2017-2023. http://www.miyagi.kopas.co.jp/JSFS/jsfs-english/E-PUB/index.html

58. Li X, Kijima, A. Gynogenetic tetraploid larvae of Pacific oyster Crassostrea gigas induced by inhibition of 1st and 2nd meiotic divisions. Tohoku Journal of Agricultural Research. 2006; 57(1-2). http://hdl.handle.net/10097/40396

59. Guo X, Hershberger W K, Cooper K, Chew K K. Tetraploid induction with mitosis l, inhibition and cell fusion in the Pacific oyster (Crassostrea gigas Thunberg). J Shellfish Res. 1994; 13:193-198. https://www.shellfish.org/jsr-public 